CERN-TH/95-256

IFUM $519 / \mathrm{FT}$

\title{
Heavy Flavour Production
}

\author{
Paolo Nason' \\ CERN TH-Division, CH-1211 Geneva 23, Switzerland
}

Stefano Frixione

Dip. di Fisica, Università di Genova, and INFN, Sezione di Genova, Genoa, Italy

Giovanni Ridolfi

CERN TH-Division, CH-1211 Geneva 23, Switzerland

\begin{abstract}
We review the status of heavy flavour production in QCD. Comparison of experimental and theoretical results for top and bottom production are given. Selected topics in charm production are also discussed.
\end{abstract}

Invited talk given at the XV International conference "Physics in Collisions" Cracow, Poland, June 8-10, 1995.

CERN-TH/95-256

IFUM 519/FT

September 1995

\footnotetext{
${ }^{1}$ On leave of absence from INFN, Sezione di Milano, Milan, Italy.

${ }^{2}$ On leave of absence from INFN, Sezione di Genova, Genoa, Italy.
} 
Although the first next-to-leading-order calculations of heavy flavour production were performed more than five years ago [1] [2], progress in this field is constantly being made. The work of ref. [3] has confirmed the results of ref. [1]. A calculation of the next-to-leading cross section for the photoproduction of heavy quarks has been given in ref. [⿴囗十 , and has been confirmed by ref. [5]. The computation of the radiative corrections to the electroproduction of heavy quarks, via an off-shell photon, was presented in ref. [6]. A method that accounts for the correlation of heavy quarks at next-to-leading order was developed in ref. [7] for heavy quark hadroproduction. An application of this calculation to fixed-target production of heavy quarks is given in ref. [8]. The method was extended to the photoproduction of heavy quarks in ref. [9].

In the following I will describe some recent experimental and theoretical progress in top, bottom and charm production. I briefly remind the reader of the QCD mechanism for heavy quark production. The hadroproduction process is depicted in fig. 1. The

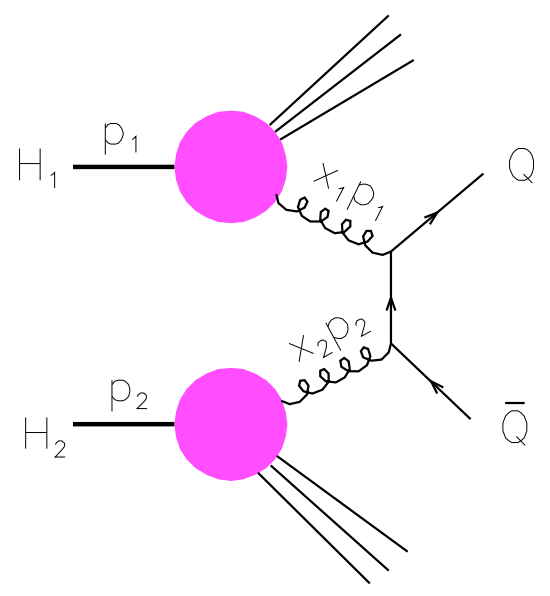

Figure 1: The heavy quark production process in perturbative QCD.

high energy colliding hadrons can be viewed as a broad band beam of partons (quarks and gluons), which collide and fuse to produce the heavy quark pair. Thus the cross section is given by the formula

$$
\sigma_{Q \bar{Q}}=\int d x_{1} d x_{2} f_{i}^{H_{1}}\left(x_{1}, \mu\right) f_{k}^{H_{2}}\left(x_{2}, \mu\right) \hat{\sigma}_{i j}\left(x_{1} p_{1}, x_{2} p_{2}, m_{Q}, \mu, \alpha_{S}\right)
$$

where $\hat{\sigma}$ is the short-distance cross section, which is calculable order by order in perturbation theory. In fig. 1 1 only one lowest order diagram is represented. Diagrams 
like the one of fig. 1, after squaring the amplitude to obtain a cross section, give rise to a contribution of order $\alpha_{S}^{2}$. Thus, the leading process is of order $\alpha_{S}^{2}$, and the next-to-leading one is $\alpha_{S}^{3}$. The coupling constant $\alpha_{S}$ is evaluated at a scale of the order of the mass of the heavy flavour. Thus, for charm the coupling constant $\alpha_{S}$ is roughly 0.3 to 0.5 , for bottom is around 0.2 , and for top is 0.1 . We expect therefore that theoretical predictions should be very reliable for top, less for bottom, and even less for charm. In fact, next-to-leading corrections are around 30-40\% for top, 100\% for bottom, and even larger and less controllable for charm. It is clear therefore that for top we expect to be able to predict the cross section rather well. For bottom, and expecially for charm, we can expect deviations from theoretical predictions due to higher order and non-perturbative effects. In these cases, we also must keep in mind whether certain effects have a simple explanation in terms of some non-perturbative effects, and whether one can model the cross section with the perturbative result supplemented with some model for the non-perturbative effects.

I will first deal with the issue of comparing the measured top cross section to theoretical expectations. I will then discuss the status of bottom production. Charm production physics is a rather wide and complex field, mostly because of the fact that non-perturbative effects in charm production do play an important role. Thus, there are several experimental issues of a certain interest, like the $A$ dependence, and the leading particle effect. Here I will focus upon what I think are the most important discrepancies between perturbation theory predictions and experimental results. I will show that certain distributions, like the transverse momentum of charmed mesons, the transverse momentum of the pair, the azimuthal distance of the pair, and the invariant mass of the pair, are somewhat in contrast with each other, and that they may give an indication of how the comparison of theory and data should be performed.

\section{Top production}

For top hadroproduction, it was found that radiative corrections are generally well under control. This allows us to make predictions for top cross sections with a relatively small error. The recent measurements of top cross section at the Tevatron [10], [11] have turned out to be in remarkable agreement with the theoretical predictions. This is illustrated in fig. 2. In the figure we have reported the result of the old calculation of ref. [12], using the DFLM structure functions, a next-to-leading 
calculation using the MRSD- set, and the calculation of ref. 113 which attempts to resummation of Sudakov threshold effects. Other calculations are reported in ref [14] [15], where in the latter an alternative approach to the inclusion of threshold effects is attempted. When estimating the cross section, one should not forget that the uncertainty on $\Lambda_{5}$, which defines the strenght of the QCD coupling constant, is larger than what is usually assumed in structure function fits. In fact, all LEP determinations [16] tend to give a larger value. Therefore, for comparison, we also show the computed cross section in case one uses a value of $\Lambda_{5}$ more compatible with LEP determination. The corresponding increase of the cross section is presumably an overestimate, since a larger value of $\Lambda_{5}$ implies stronger evolution of the parton densities, which soften more rapidly as the momentum scale increases. The figure shows that only minor changes in the theoretical predictions have taken place in the last few years. We can expect that in spite of the fact that more subleading contributions may be added in the future to the top strong cross section, they will not change substantially the pure next-to-leading result.

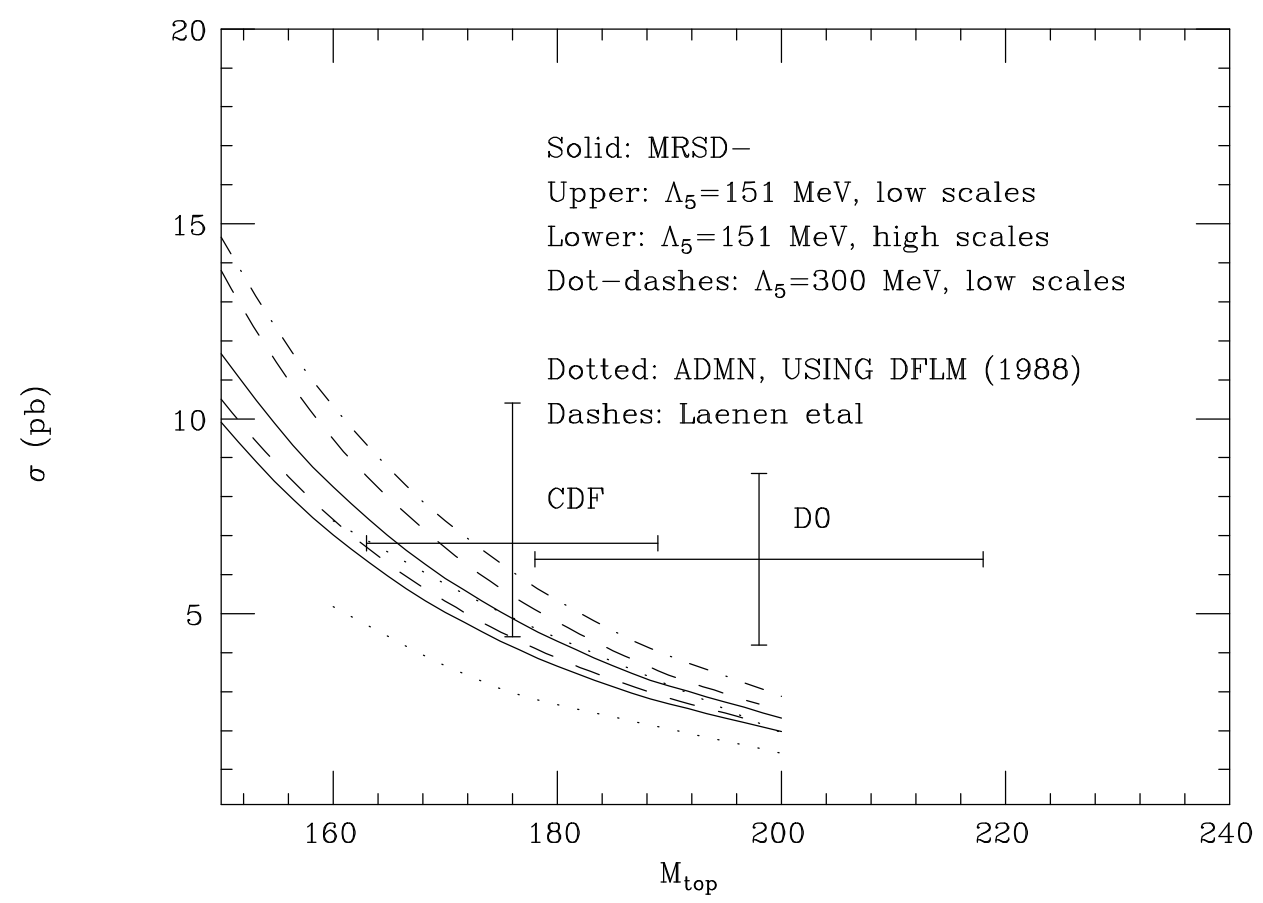

Figure 2: Cross sections for top production: various calculations versus the CDF and D0 measurements. 


\section{Bottom production}

In bottom production radiative corrections are large, and various estimates of corrections of even higher order (as given for example by the renormalization and factorization scale dependence) lead to theoretical uncertainties of the order of a factor of 2. These uncertainties, when combined with other physical uncertainties, such as the error in the knowledge of $\Lambda_{\mathrm{QCD}}$ and of the structure functions, result in a rather poor theoretical prediction. The CDF [17], D0 [18] and UA1 [19] experiments have all measurements of the spectrum of $\mathrm{B}$ mesons. The earliest CDF measurements reported a cross section which was much higher than QCD prediction, and seemed to be in contrast with CDF measurements. This problem was due to the poor theoretical understanding of the direct $J / \Psi$ production, and after the introduction of microvertexing techniques, the cross sections have come down to smaller values. The remaining discrepancy which is often quoted in CDF publications is due to the fact that modern sets of structure functions tend to favour small values for $\Lambda_{5}$, and therefore smaller cross sections. If we instead allow for larger values of $\Lambda_{5}$, as favoured by LEP experiments, all we can conclude is that all data (including UA1 data) are consistent with the theoretical prediction, although on the high side of the theoretical band. What once seemed to be a discrepancy between CDF and UA1 data, is now gone, both data sets bearing the same relation with respect to the theoretical curves. This is illustrated in fig. 3. The main band (solid) is obtained using the MRSA parton densities, except that for the upper line the value of $\Lambda_{5}$ is taken to be 300 $\mathrm{MeV}$, not quite consistent with DIS data, but closer to what is indicated by LEP measurements. The same curves are also shown without fragmentation effects, in comparison with the full band (dotted lines) obtained interpolating table 6 of ref. [2]. As one can see not much has changed since then in the theoretical prediction, and the only thing one can say is that the CDF measurement is closer to the upper limit of the theoretical band. For comparison, I also show data from the D0 collaboration in fig. 4 . The old UA1 data is shown in fig. 5. When comparing theoretical and experimental curves, one should remember that certain experimental results are deconvoluted from the heavy quark fragmentation effects, and are given as a bare quark cross section, while others are heavy flavour meson's cross sections. The former should be compared to the theoretical prediction obtained without the inclusion of fragmentation effects, while for the latter one should include fragmentation. The UA1 experiment presented both a $B$ hadron cross section and a $b$ quark cross section, by deconvoluting the effect 
of fragmentation. In the figure we show both the predicitons of ref. [2], and a more modern one, performed using the MRSA structure functions, and the same variation of parameters we used at Tevatron energy. Again, we see that the theoretical band has not changed much. The UA1 quark data is on the high side of the theoretical band, as the CDF and D0 data. This fact has been quantified in the study of ref. [20], where it is found that the ratio of the data over theoretical upper band is 0.97 for D0, 1.3 for CDF, and 0.84 for UA1.

We can conclude by saying that data on inclusive $b$ production at hadron colliders is consistent with QCD expectation, and lies on the upper extreme of the prediction band. In other words, in order to fit the cross section, one is forced to use values of the parameters (like the scales, the mass, and the value of $\Lambda$ ) that favour higher cross section.

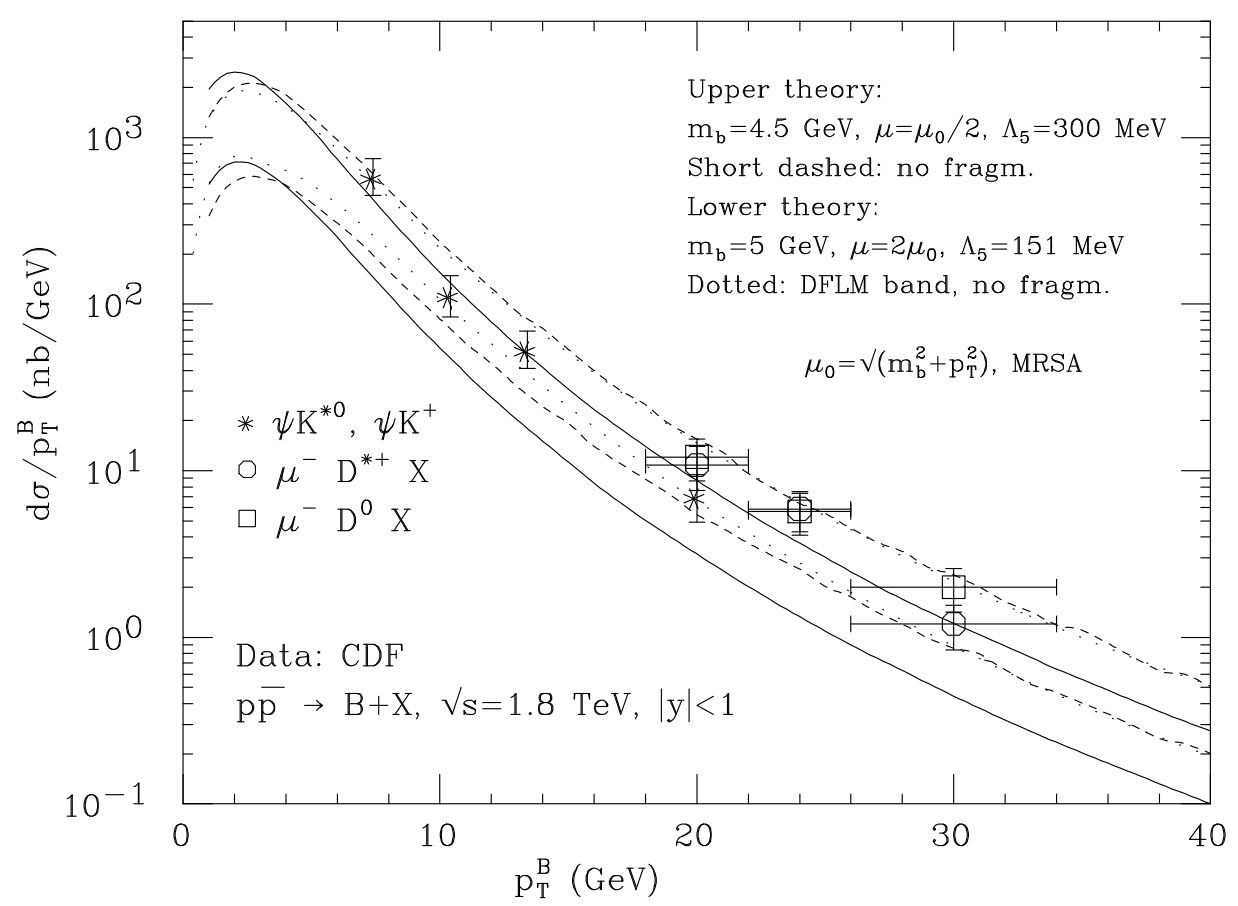

Figure 3: Transverse momentum spectrum of B mesons at Tevatron energies, as measured by the CDF collaboration. The main prediction band (solid lines) is also shown without the inclusion of fragmentation effects. The dotted lines are the old prediction of ref., using the DFLM structure functions sets. 
As first pointed out in ref. [1], the perturbative computation of the $b$ cross section at hadron colliders reaches a difficult kinematical regime when going from the $\mathrm{Sp} \overline{\mathrm{p}} \mathrm{S}$ energies to the Tevatron. This is due to the appearance of large logarithms of $S / \mathrm{m}^{2}$ (where $S$ is the c.m. energy squared, and $m$ is the mass of the heavy quark being produced) in the perturbative expansion. In other words, at very large $S$ the expansion parameter becomes $\alpha_{S} \log \left(S / m^{2}\right)$, which (for $S$ large enough) becomes of order 1 , thereby spoiling the convergence of the perturbative expansion. Quite substantial theoretical work was performed on the small- $x$ problem [21]. In particular, in ref. [22] it was found that the resummation of small- $x$ effects increases the total rate by $30 \%$. These effects are very likely to operate in the small $p_{t}$ region. It is unlikely, however, that they would strongly improve the agreement between theory and data.

More detailed analysis of bottom distributions have been performed at the Tevatron. In refs. [23] the azimuthal correlation has been measured and compared with the theoretical prediction of ref. [7]. While in qualitative agreement with QCD prediction, important differences are observed. We can hope that correlation measurements

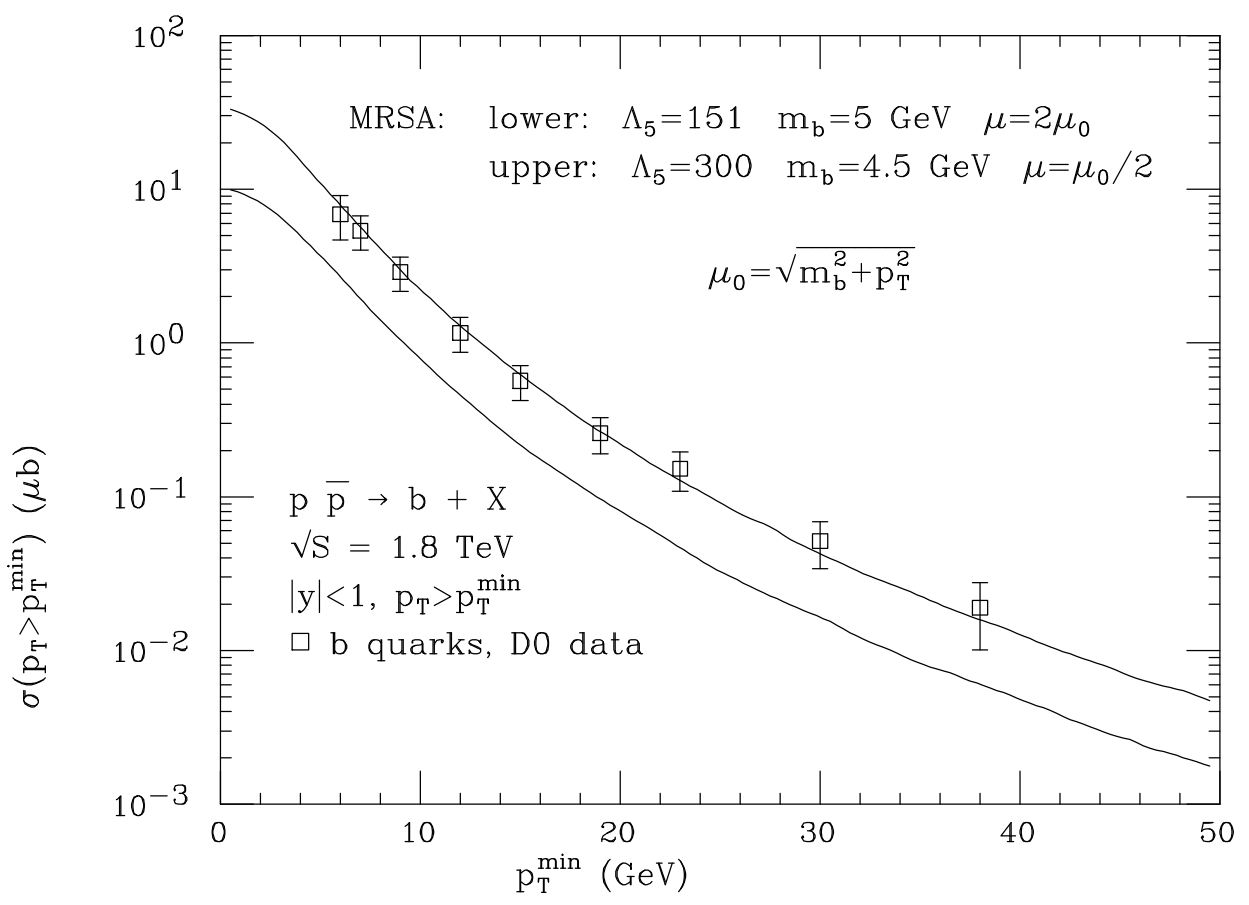

Figure 4: Cross section for $b$ quark production as a function of a transverse momentum cut, as measured by the D0 collaboration. 
of this kind may shed a light also on the problems observed in the single inclusive $p_{T}$ distribution.

\section{Charm production}

Figure 6 gives an instructive picture of the uncertainties in charm and bottom cross sections at fixed-target experiments. Observe the considerable improvement that takes place when going from charm to bottom. Observe also the strong mass dependence of the charm result. Needless to say, similar uncertainties plague the $p N$ cross sections, as shown in fig. 7. The experimental measurement of bottom production cross section in proton-nucleon collisions at fixed target is a new result [24. New-generation fixed-target experiments have accumulated very large statistics of charm events. For a (possibly incomplete) list of recently published results, see refs. [25] and [26]. For recent reviews of the current experimental situation and future

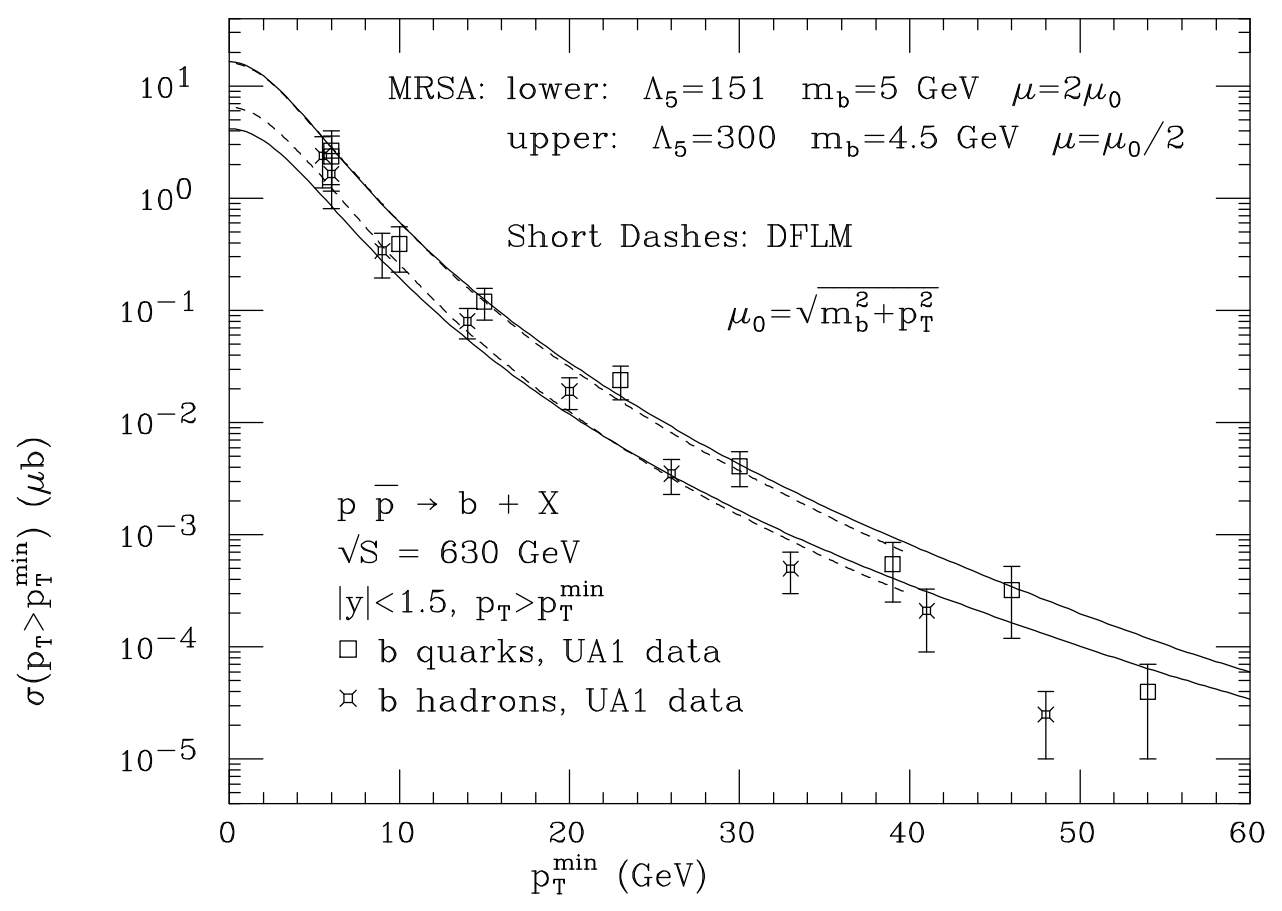

Figure 5: Transverse momentum spectrum of B mesons at $630 \mathrm{GeV}$ center of mass energy, measured by the UA1 collaboration. 
perspectives, see refs. [27], [28]. For an older review see [29]. Results on bottom production at fixed target are given in refs. 30]-31. As one can see, experimental results on total cross sections for charm and bottom production at fixed target are in reasonable agreement with theoretical expectations. We remind the reader that many puzzling ISR results in $p p$ collisions at $62 \mathrm{GeV}$ remain difficult to explain (see the review [29]), in particular the large $\Lambda_{b}$ production rates reported in ref. [31].

Photoproduction results are also in fairly good agreement with theory, as can be seen from fig. 8. The large band in the figure is obtained by varying all parameters, including a variation of the charm quark mass from 1.2 to $1.8 \mathrm{GeV}$. The dashed and dotted bands are instead obtained by varying all other parameters, and keeping the mass of the charm quark fixed to $1.5 \mathrm{GeV}$. Very recently a new data point has been added by HERA [32]. At the Bruxelles conference a new H1 result has been announced [33].

Differential distributions for charm are at present in a more complex situation. It

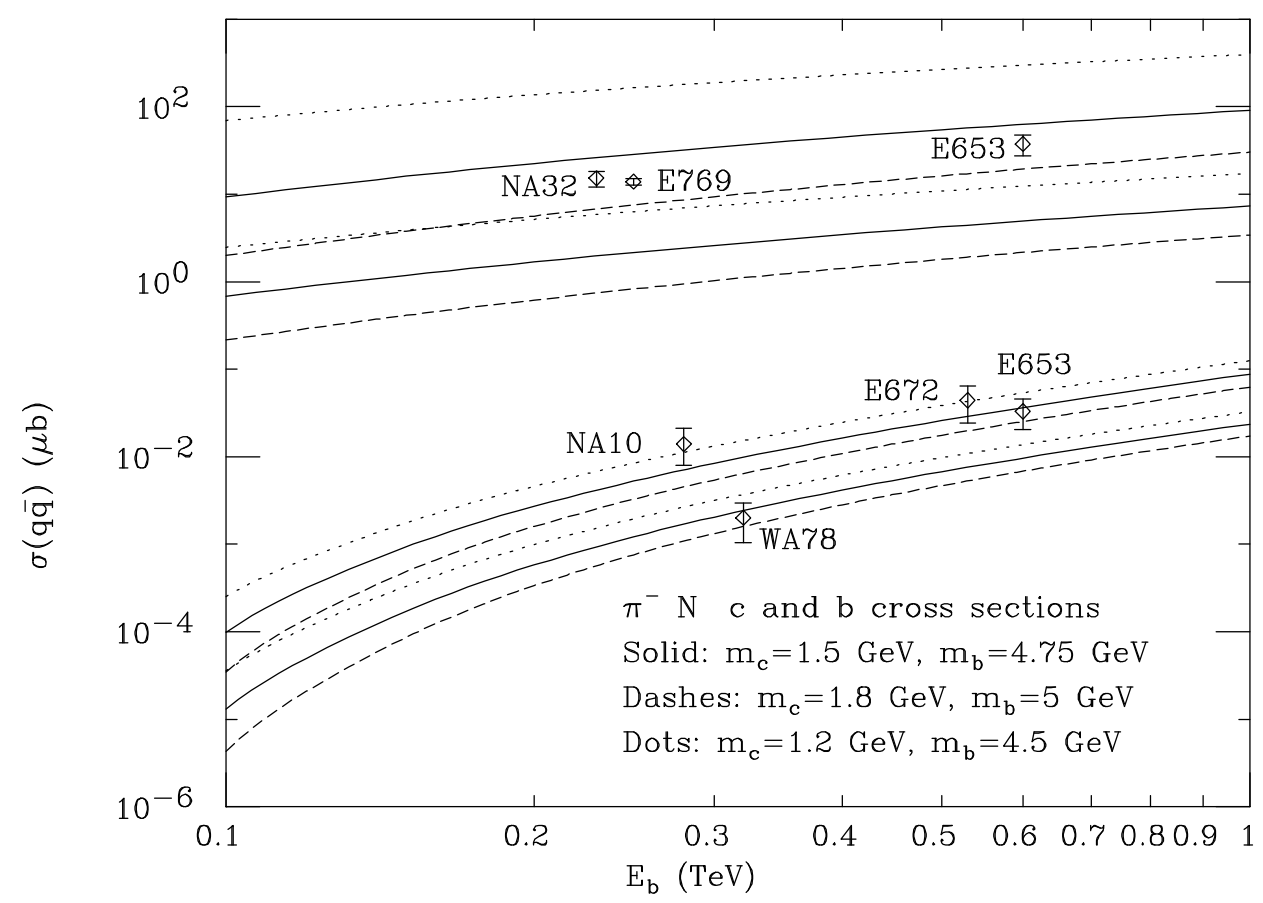

Figure 6: Cross sections for $b$ and $c$ production in $\pi N$ collisions versus experimental results. 
turns out that in photoproduction one finds a remarkably good agreement between theoretical expectations and experiments. One typically looks at the transverse momentum distribution of a single quark, the transverse momentum of the pair, the invariant mass and the azimuthal correlation of the pair. All this quantities are in good agreement with theoretical expectations, provided one includes in the calculation the effect of a fragmentation function, parametrized in the same way as in charm production in $e^{+} e^{-}$collisions. This is not the case in hadroproduction. Typically one sees that the inclusive $p_{t}$ distribution of a single meson is well described by pure QCD, without the inclusion of a fragmentation function. One also sees a similar behaviour for the $x_{f}$ distributions. The azimuthal correlation of charmed pairs requires the inclusion of some non-perturbative effects, that could be described as a primordial transverse momentum of the incoming quarks. This transverse momentum turns out to be of reasonable size, that is to say, below $1 \mathrm{GeV}^{2}$. Another observable which is strongly sensitive to a primordial transverse momentum of the incoming quarks is the transverse momentum distribution of the charmed pair. In this case, only a very

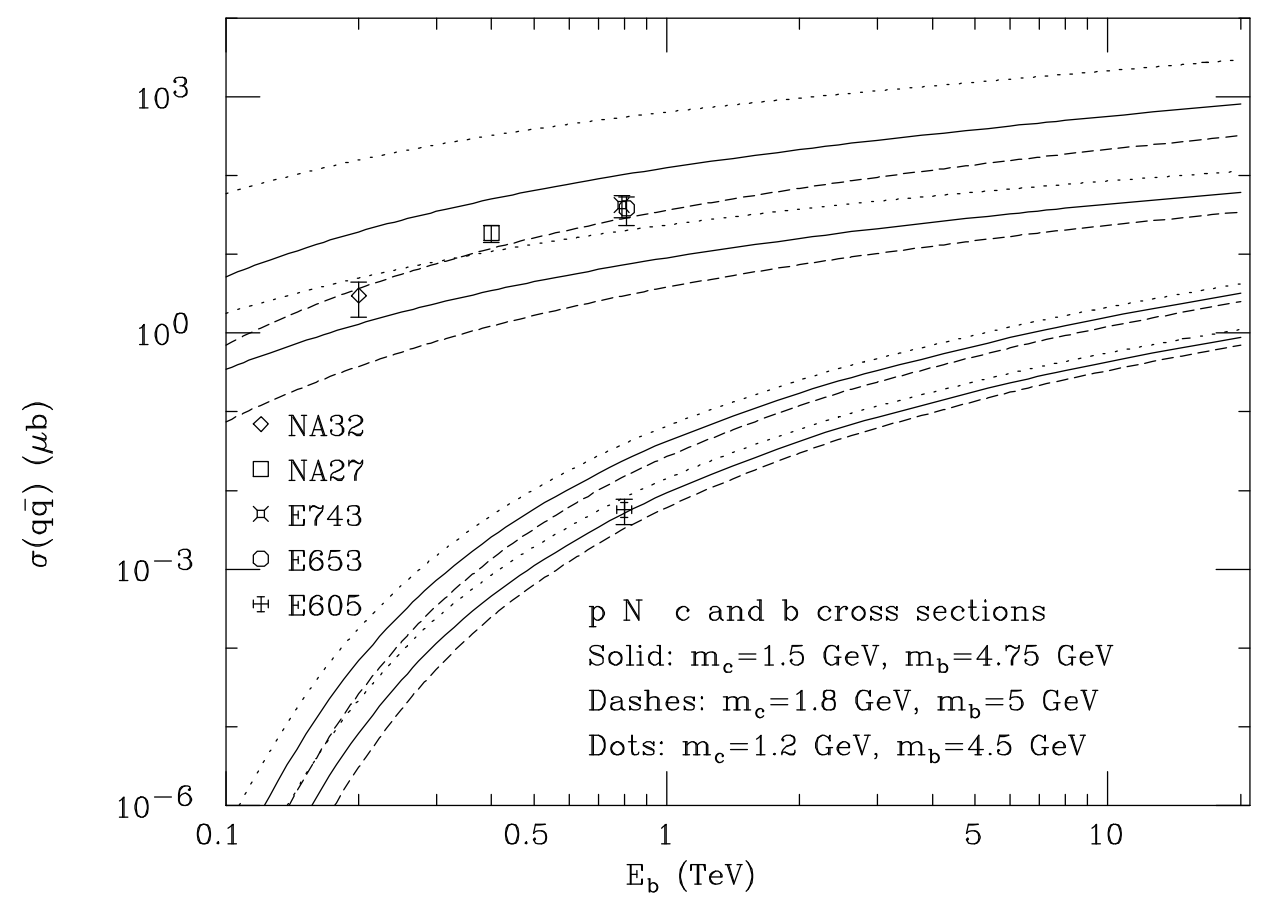

Figure 7: Cross sections for $b$ and $c$ production in $p N$ collisions versus experimental results. 
large primordial transverse momentum could reproduce the measured cross section. In the following I will illustrate this problems. It is fair to say that a satisfactory answer to these problems is not known yet, but also that present data gives some hints for a direction in which to deepen theoretical work. I begin by showing in fig. 9 the azimuthal distance between the charm and anticharm, taken from ref. [34]. It is quite obvious that a strong enhancement in the back-to-back region is observed, as expected from a hard production mechanism. Perturbative calculations display a similar behaviour, although they are generally much more peaked, as shown in fig. 10. The solid histogram in the figure is obtain by a purely perturbative calculation. The dashed and dotted histogram are obtained by assuming that incoming partons do have some non-vanishing transverse momentum, which is transmitted to the final quark-antiquark pair. This transverse momentum can be viewed as a simple-minded model for non-perturbative effects, due to the fact that hadrons have a finite transverse size. The figure was obtained for a beam energy of $230 \mathrm{GeV}$, but for the sake of the following discussion it can be considered as energy independent. We see that

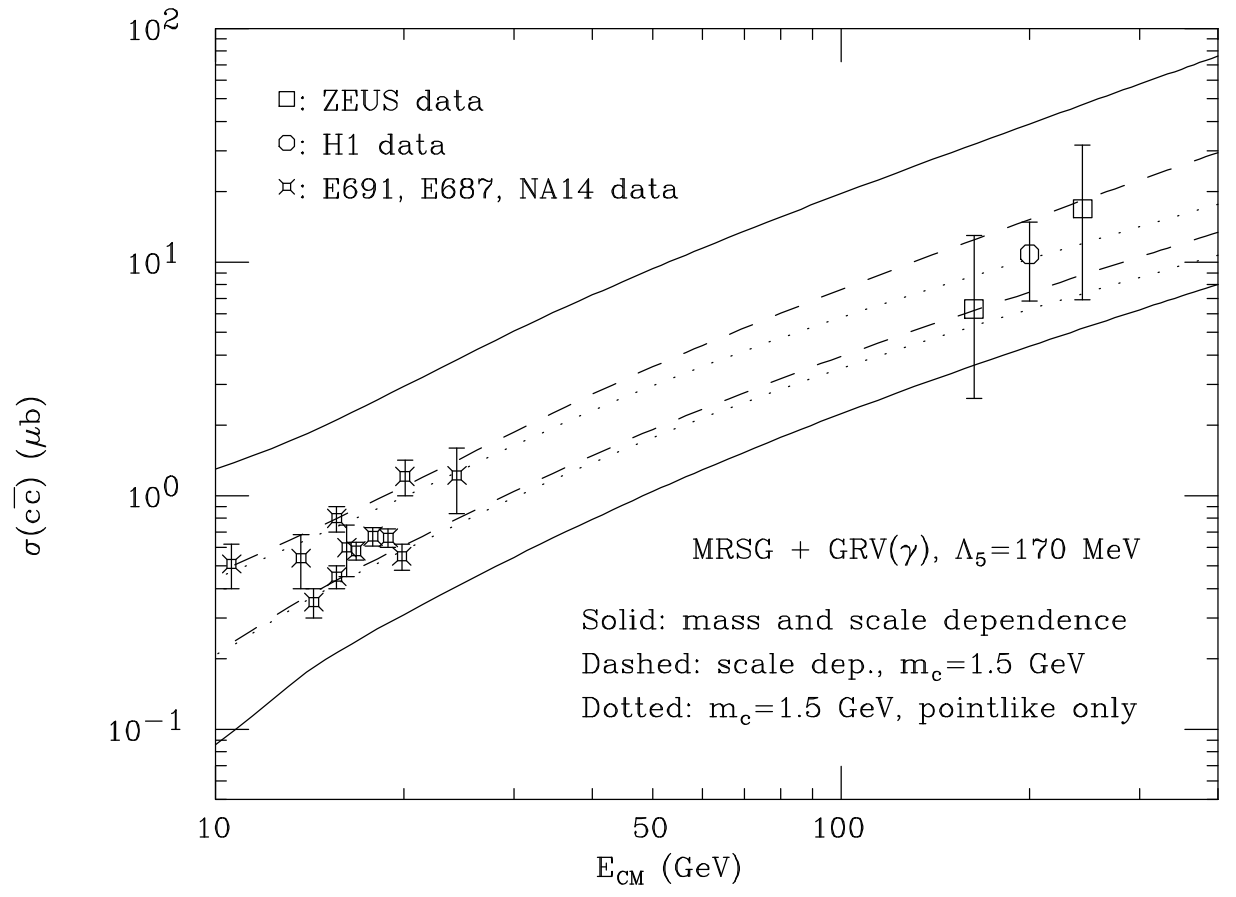

Figure 8: Cross sections for $c$ production in $\gamma N$ and $\gamma p$ collisions versus experimental results. 
all data seems to favour a $\left\langle k_{T}^{2}\right\rangle$ not larger than $1 \mathrm{GeV}^{2}$, the WA92 data favouring even smaller values. A value around $1 \mathrm{GeV}^{2}$ is roughly what one would expect, based upon experience with Drell-Yan pair production, which requires intrinsic transverse momenta of the order of $600 \mathrm{MeV}^{2}$. Drell-Yan pairs are mainly produced via quarkantiquark annihilation, while heavy flavours are mostly produced by gluons. Since the gluons couple more strongly than quarks by a factor of $9 / 4$, one expects that the associated transverse momentum should be somewhat larger. Observe that the azimuthal correlation is independent of fragmentation effets, since fragmentation does not change the direction of the outgoing particle. In fig. 9, data from the E687 pho-
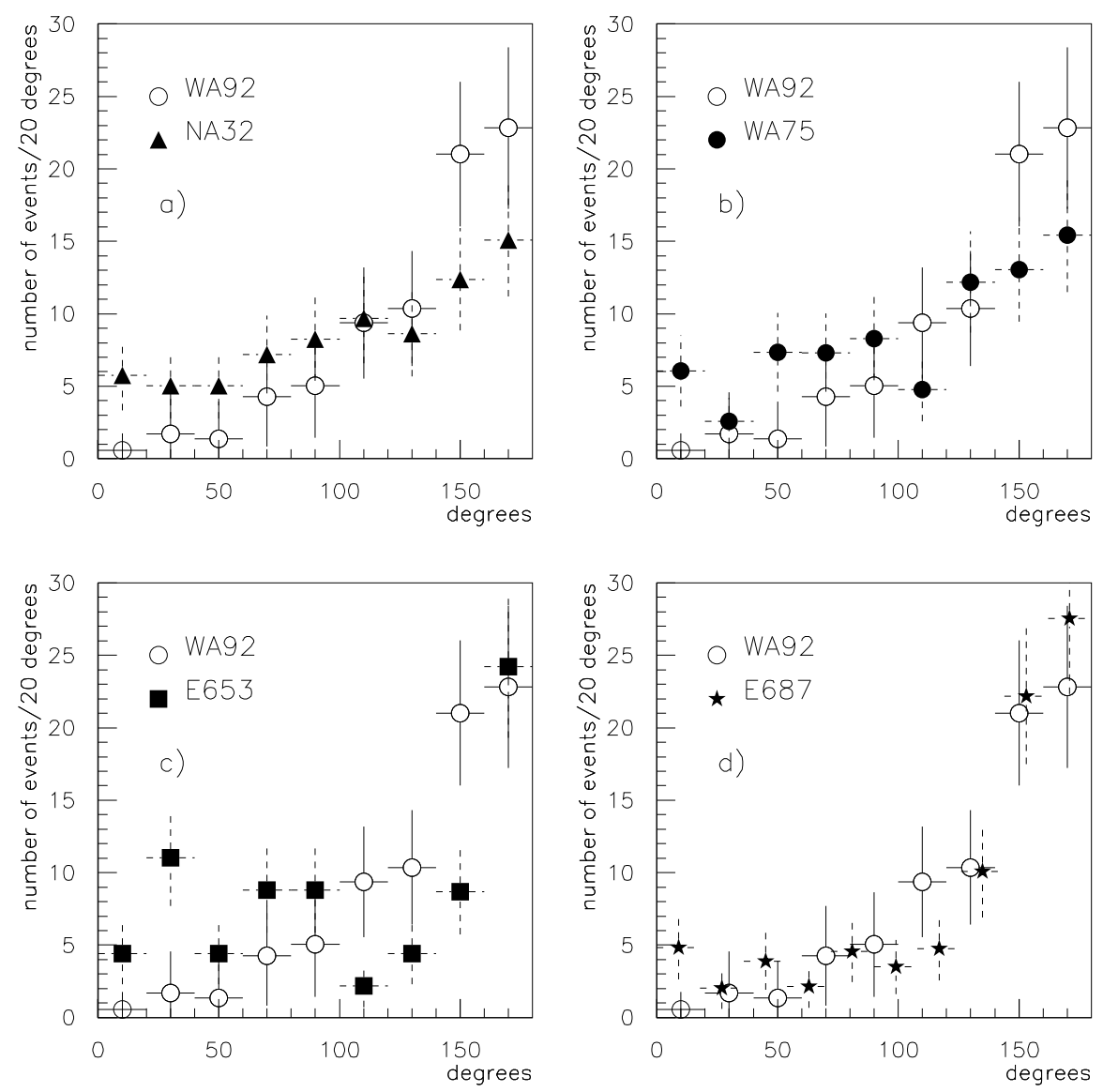

$\Delta \varphi$

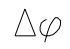

Figure 9: Azimuthal correlation in the production of charmed pairs. 
toproduction experiment is also reported [35]. Comparisons of the E687 data with theoretical expectation are given in ref. [9], and a good agreement is found, whether or not one adds an intrinsic momentum kick to the incoming parton. In fact, in the case of photoproduction, a transverse momentum kick has a less dramatic effect, since only one parton is carrying the kick, and also because the perturbative distribution is already broad, and it washes out the effect of the kick.

Let us now turn to the inclusive transverse momentum distributions. Here we find a problem in the comparison of photoproduction and hadropoduction data, as illustrated in figs. 11 and 12 . We see that photoproduction data is in good agreement with QCD predictions, including the effects of fragmentation. Transverse momentum kicks do not alter the distribution in an appreciable way. In the case of hadroproduction, one finds instead that the perturbative prediction is in good agreement with data, and that the inclusion of fragmentation effects spoil the agreement. In the figure, also a transverse momentum kick is included, which improves the agreement, but we still see that even a $1 \mathrm{GeV}^{2}$ primordial transverse momentum is not enough

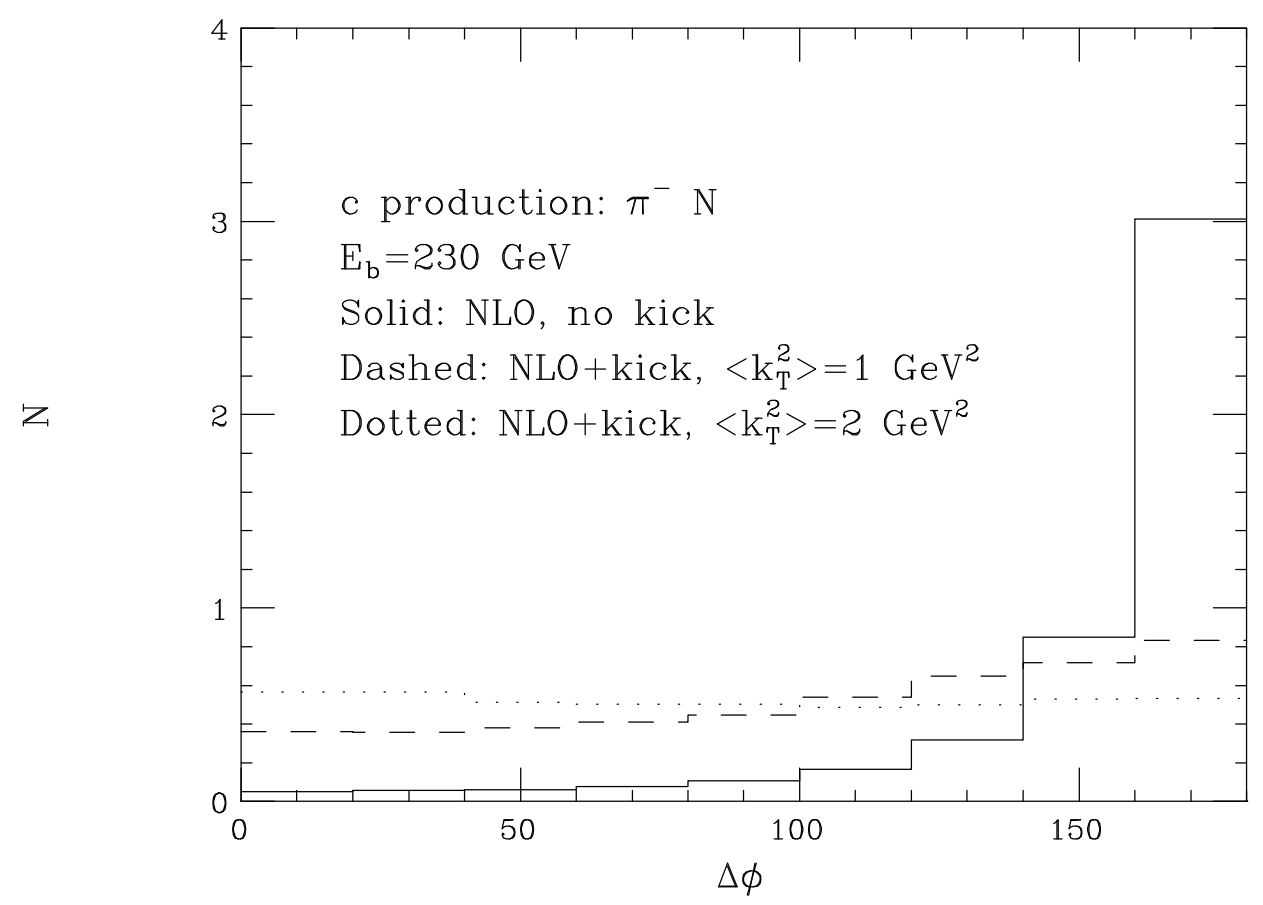

Figure 10: Theoretical predictions for the azimuthal correlation in the production of charmed pairs. 
to overcome the effect of fragmentation.

A similar situation arises in the distributions of the transverse momentum of the charmed pair. As an example I report in table 1 a computation of various average quantities, in pion-nucleon collisions at $230 \mathrm{GeV}$, in comparison with the recent study of ref. [36]. These results deserve a comment. We see that, aside from the rapidity difference, both the transverse momentum of the pair and the invariant mass of the pair would be in better agreement with the data if the effect of fragmentation was much less dramatic. In fact, if we don't include fragmentation effects at all, we see that all transverse distributions are in good agreement with theoretical prediction, provided one is willing to accept the possibility of a non-perturbative intrinsic transverse momentum effect of the incoming parton of the order of $1 \mathrm{GeV}^{2}$, which is a very reasonable value. It is often claimed that the fact that longitudinal distributions (like the $x_{F}$ distributions) are harder than theoretical predictions may require the inclusion of higher twist effects [37]. On the other hand, the problem is there only if one insists in using the fragmentation functions for longitudinal distributions, a

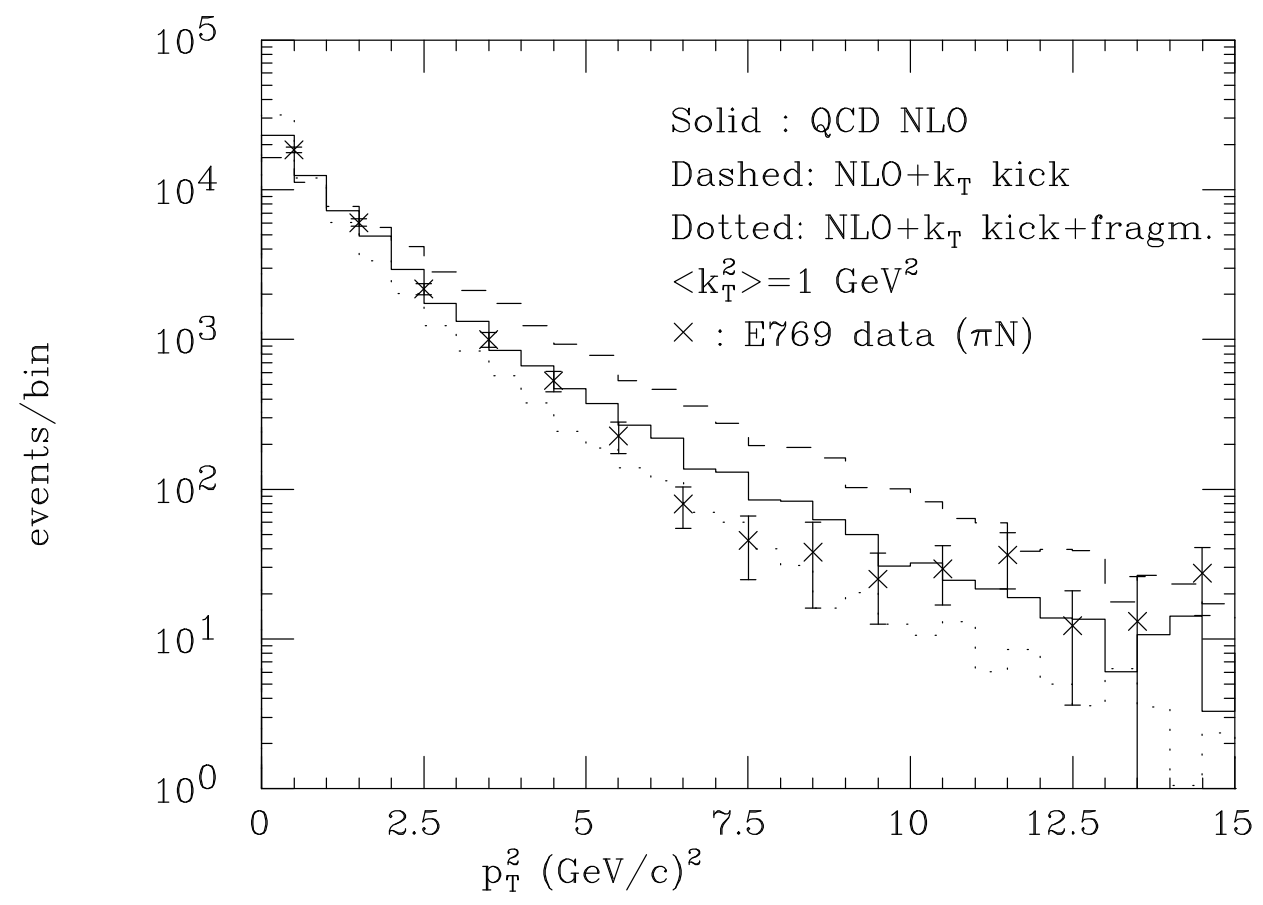

Figure 11: Single inclusive $p_{T}^{2}$ distribution measured by the E769 experiment, compared to theoretical expectations. 
procedure that is not fully justified. Many models are capable of justifying easily the observed longitudinal distributions. On the contrary, the fact that transverse momentum distributions are harder than expected is much more worrying, since in this case the factorization theorem should apply. One possible way out of this problem

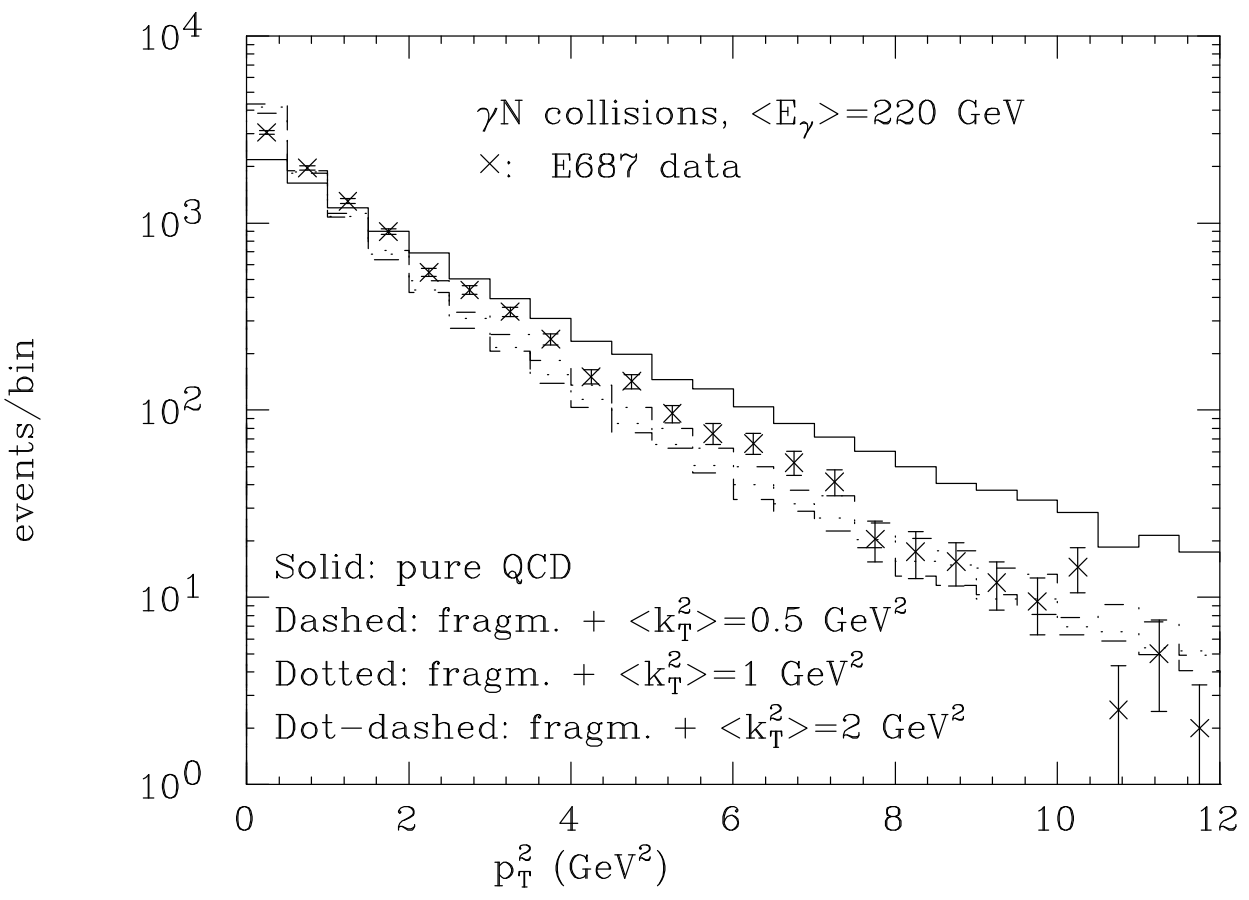

Figure 12: Single inclusive $p_{T}^{2}$ distribution measured by the E687 experiment, compared to theoretical expectations.

\begin{tabular}{|l||c|c|c|c|c|c|}
\hline \multicolumn{1}{|c||}{} & bare & \multicolumn{2}{c|}{$\left\langle k_{T}^{2}\right\rangle=1 \mathrm{GeV}^{2}$} & \multicolumn{2}{c|}{$\left\langle k_{T}^{2}\right\rangle=2 \mathrm{GeV}^{2}$} & data \\
& & no fragm. & with fragm. & no fragm. & with fragm. & \\
\hline$\left\langle p_{Q \bar{Q}}^{2}\right\rangle$ & 0.277 & 2.24 & 1.116 & 4.2 & 2.04 & $1.98 \pm 0.11 \pm 0.09$ \\
\hline$\left\langle M_{Q \bar{Q}}\right\rangle$ & 4.20 & 4.20 & 3.62 & 4.20 & 3.63 & $4.45 \pm 0.03 \pm 0.13$ \\
\hline$\left\langle\Delta y_{Q \bar{Q}}\right\rangle$ & 0.931 & 0.886 & 0.669 & 0.852 & 0.650 & $0.54 \pm 0.02 \pm 0.24$ \\
\hline
\end{tabular}

Table 1: Various average quantities computed with and without fragmentation effects, and transverse momentum kick of 1 and $2 \mathrm{GeV}^{2}$, compared with the results of the data analysis of ref. 36. 
may be related to the fact that a large fraction of bottom cross section in hadroproduction comes from gluon fragmentation into heavy quarks, while in the case of $e^{+} e^{-}$ annihilation, and also in the case of photoproduction, this fraction is much smaller. This possibility requires further study, but it could also explain why collider data for bottom production is on the high side of the prediction band. We have in fact seen that also in that case, if the fragmentation function is not included, the agreement between theory and data becomes much better.

\section{References}

[1] P. Nason, S. Dawson and R. K. Ellis, Nucl. Phys. B303(1988)607

[2] P. Nason, S. Dawson and R. K. Ellis, Nucl. Phys. B327(1988)49.

[3] W. Beenakker et al., Stony Brook preprint ITP-SB-90-46 (1990); Phys. Rev. D40(1989) 54 .

[4] R.K. Ellis and P. Nason, Nucl. Phys. B312(1989)551.

[5] J. Smith and W.L. Van Neerven, Nucl. Phys. B374(1992)36.

[6] E. Laenen, S. Riemersma, J. Smith and W.L. Van Neerven, Nucl. Phys. B392(1993)162 and 229.

[7] M.L. Mangano, P. Nason and G. Ridolfi, Nucl. Phys. B373(1992)295.

[8] M. Mangano, P. Nason and G. Ridolfi, Nucl. Phys. B405(1993)507.

[9] S. Frixione, M. L. Mangano, P. Nason and G. Ridolfi, Nucl. Phys. B412(1994)225.

[10] F. Abe et al., CDF Coll., Phys. Rev. Lett. 74(1995)2626.

[11] S. Abachi et al., D0 Coll., Phys. Rev. Lett. 74(1995)2632.

[12] G. Altarelli, M. Diemoz, G. Martinelli and P. Nason, Nucl. Phys. B308(1988)607.

[13] E. Laenen, J. Smith and W.L. van Neerven, Nucl. Phys. B369(1992)543;

Phys. Lett. 321B(1994)254. 
[14] R.K. Ellis, Phys. Lett. B259(1991)492.

[15] E.L. Berger and H. Contopanagos, preprint ANL-HEP-PR-95-31, hepph/9507363.

[16] M. Schmelling, this proceedings (also CERN-PPE/95-129).

[17] F. Abe et al., CDF Coll., preprint FERMILAB-Pub-95/48-E, March 18, 1995; F. Abe et al., CDF Coll., Phys. Rev. Lett. 68(1992)3403; 69(1992)3704; 71(1993)500, 2396 and 2537.

[18] D0 Collaboration, S. Abachi et al., Phys. Rev. Lett. 74(1995)3548.

[19] UA1 collaboration, Phys. Lett. B256(1991)121.

[20] M.L. Mangano, preprint CERN-TH/95-191, hep-ph/9508260, to appear in the Proceedings of the 6th International Symposium on Heavy Flavour Physics, Pisa, Italy, June 6-10, 1995.

[21] R.K. Ellis and D.A. Ross, Nucl. Phys. B345(1990)79;

S. Catani, M. Ciafaloni and F. Hautmann, Phys. Lett. B242(1990)97, Nucl. Phys. B366(1991)135, Nucl. Phys. B (Proc. Suppl.) 23B(1991)328.

[22] J.C. Collins and R.K. Ellis, Nucl. Phys. B360(1991)3.

[23] F. Abe et al., CDF Coll., preprint FERMILAB-PUB-95/289-E, hep-ex9508017;

F. Abe et al., CDF Coll., preprint FERMILAB-PUB-94/131-E.

[24] D.M. Jansen et al., E605 Coll., Phys. Rev. Lett. 74(1995)3118.

[25] K. Kodama et al., E653 Coll., Phys. Lett. B284(1992)461;

S. Aoki et al., WA75 Coll., Prog. Theor. Phys. 87(1992)1305;

S. Barlag et al. NA32 Coll., Z. Phys. C49(1991)555;

R. Ammar et al., E743 Coll., Phys. Rev. Lett. 61(1988)2185.

[26] M. Aguilar-Benitez et al., NA27 Coll., Phys. Lett. 164B(1985)404; Z. Phys. C40(1988)321;

S. Aoki et al., WA75 Coll., Phys. Lett. 209B(1988)113; Prog. Theor. Phys. 87 (1992) 1315; 
K. Kodama et al., E653 Coll., Phys. Lett. 263B(1991)579; CMU-HEP9118(1991);

S. Barlag et al., NA32 Coll., Phys. Lett. 257B(1991)519.

[27] J.A. Appel, Annu. Rev. Nucl. Part. Sci. 42(1992)367;

L. Rossi, preprint INFN-AE-91-16, Oct. 1991, presented at 4th Int. Symp. on Heavy Flavour Physics, Orsay, France, 25-29 June 1991.

[28] G. Introzzi, E771 Coll., 3rd Topical Seminar on Heavy Flavours, San Miniato 17-21 June 1991, to appear in Nucl. Phys. B.

[29] S.P.K. Tavernier, Reports on Progress in Physics 50(1987)1439.

[30] J. P. Albanese et al. (WA75 Coll.), Phys. Lett. 108B(1982)361.

[31] G. Bari et al., Nuovo Cimento 104A(1991)1787;

M. Basile et al., Nuovo Cimento 65A(1981)391.

[32] The ZEUS Collaboration, preprint DESY 95-013, hep-ex-9502002.

[33] "Photoproduction of $D^{* \pm}$ mesons in electron-proton collision at HERA", H1 collaboration, 1995 International Europhysics Conference on High Energy Physics, $27 / 7$ to $2 / 8 / 1995$, Brussels, Belgium.

[34] The BEATRICE COLLABORATION, preprint CERN/PPE 94-214, DEC. 1994.

[35] M.P. Alvarez et al. (NA14/2 Coll.), Phys. Lett. B278(1992)385, V. Arena et al. (E687 Coll.), Phys. Lett. B308(1993)194;

J.C. Anjos et al. (E691 Coll.), Phys. Rev. Lett. 65(1990)2503;

M.I. Adamovich et al. (Photon Emulsion Coll.), Phys. Lett. B187(1987)437 and references therein.

[36] K. Rybicki and R. Ryłko, Preprint BRU/PH/201, hep-ex-9505005.

[37] S.J. Brodsky, P. Hoyer, A.H. Mueller and W. Tang, Nucl. Phys. B369(1992)519. 